\title{
Urgences
}

\section{L'embrumé de Viard et Zacharias : Hamlet de Shakespeare en roman policier}

\section{Vianney Gallant}

Numéro 25, octobre 1989

Multiples de Hamlet

URI : https://id.erudit.org/iderudit/025543ar

DOI : https://doi.org/10.7202/025543ar

Aller au sommaire du numéro

Éditeur(s)

Urgences

ISSN

0226-9554 (imprimé)

1927-3924 (numérique)

Découvrir la revue

Citer cet article

Gallant, V. (1989). L'embrumé de Viard et Zacharias : Hamlet de Shakespeare en roman policier. Urgences, (25), 41-53. https://doi.org/10.7202/025543ar d'utilisation que vous pouvez consulter en ligne.

https://apropos.erudit.org/fr/usagers/politique-dutilisation/ 


\section{L'embrumé de Viard et Zacharias: Hamlet de Shakespeare en roman policier \\ Vianney Gallant}

Transformer un texte initial, faire advenir un hypertexte, c'est faire une lecture de cet hypotexte ou texte initial ${ }^{1}$. L'enquête policière qui encadre l'action transposée du Hamlet de Shakespeare, dans L'embrume ${ }^{2}$ de Viard et Zacharias, s'affiche donc comme un autre degré de lecture.

Autre degré de la lecture qui se traduit en mode d'emploi spécifique de la modernisation diégétique, la transmodalisation intermodale (ici: le passage du texte théâtral au texte narratif). Celle-ci supporte et est supportée par une large transformation pragmatique dont la fonction "proximisante" ne peut-être mise en doute: le scénario policier, dont on sait la vogue contemporaine. Une enquête augure déjà une lecture, voire une

1 Gérard Genette, Palimpsestes, Paris, Seuil, coll. « Poétique ", 1982, 467 p.

2 Viard et Zacharias, L'embrumé, Paris, Gallimard, coll. "Carré noir ", n 340, $1980,185 \mathrm{p}$.

Sur le mode de la parodie, L'embrumé de Viard et Zacharias puise chez Hamlet de Shakespeare un scénario, des patronymes, des toponymes, etc. Par exemple, en vertu d'un contrat établi au départ par le paratexte, Elseneur, le lieu, devient Elsen, nom d'une famille richissime. De la pièce dont il s'inspire, L'embrumé garde des traces theâtrales: liste des personnages et des a comédiens " qui tiennent les rôles, épigraphes hamlétiennes en début de chapitre, mise(s) en abyme, tout en adoptant la structure narrative du roman policier.

Trois ans après la disparition (la noyade présumée) d'Henri Elsen, son fils Henri (Kiki) réussit, par mille stratagèmes (lettres anonymes, comportements délinquants et, par la suite, crime qui se veut parfait), à remettre l'affaire au jour. Claude Elsen (frère du disparu qui a épousé la mère de Kiki, comme il se doit) et Pierre-Paul Pollon, son complice, devront affronter la trismégiste et savante vengeance de Henri Elsen junior.

Le psychiatre (Laurat, pour Horatio) fait office d'ami. Le spectre se manifeste par téléphone; le trucage d'un odomètre de voiture permet à Kiki de renforcer ses alibis, roman policier oblige. Tous les moyens techniques sont bons pour métamorphoser Hamlet en une fresque dans laquelle les auteurs auront éminemment injecté, tramé des éléments intertextuels évocateurs, fins clins d'œil à la modernité: : Je vais être à Landru ce que Picasso est à Vélasquez. " (p. 53)

L'hécatombe rocambolesque de la fin n'échappe pas à la démesure shakespearienne. La volonté de vengeance du spectre est satisfaite; pourtant, Henri Elsen junior précède son père dans la mort, succombant lui aussi, comme tout le monde, sous les balles. C'est dans une lettre au commissaire Voltmand que le père vengeur annoncera son suicide. Pour achever la besogne. 
mise en scène, une focalisation qui subsume dans ce cas-ci les diverses opérations assumées par les auteurs en regard de leur hypotexte. Cette enquête, bien sûr, aurait pu être située au Moyen Âge comme c'est le cas dans Le nom de la rose d'Umberto Eco: mais il n'empêche que le genre "roman policier" demeure plutôt contemporain; c'est en ce sens qu'il est " proximisant" ${ }^{3}$.

L'enquête recèle des éléments de la transdiégétisation: changement de nationalité, de langue, translation spatiale du Danemark à la France, introduction d'objets modernes (revolver, téléphone, etc.). Mais on pourrait tout aussi bien inverser la proposition: ces transformations diégétiques relèvent de l'enquête policière. Le jeu de Kiki, dans la planification de ses trois séries de meurtres, constitue un jeu de cache-cache avec les experts de la justice et ne devient virtuellement son art, le mot est de lui, qu'en fonction du regard extérieur, le sien comme celui des autres, porté sur ses propres gestes. C'est peut-être là que s'effectuent les épousailles du texte source et de l'hypertexte: l'hyperpersonnage, modernisé, joue selon le scénario policier, il l'engendre et le manipule. A ce niveau, la trans-diégétisation suscite une nouvelle catégorie d'actants spectateurs et une nouvelle action: tout le mouvement propre à l'hypertexte, dont les protagonistes sont les inspecteurs et les commissaires de police.

L'enquête policière accompagne donc cette modernisation diégétique issue de la valorisation secondaire des Voltimand, Cornélius, Francisco et Bernardo, devenus, pour la cause de Viard et Zacharias, les Volmand, Cornil, Cisco et cie. Mais le scénario policier provient aussi (sans doute) de la dévalorisation (sur le plan axiologique) de Hamlet métamorphosé en Kiki/Henri Elsen junior, le criminel de facto de L'embrumé.

Si un palimpseste est déjà une lecture, une interprétation d'une œuvre préexistante, et si l'enquête constitue en ellemême un effort pour assurer la lisibilité d'événements crimi-

3 Voici une nuance plutót qu'un désaveu du constat de Genette. « La transformation pragmatique [...] est en revanche un élément indispensable, ou plutôt une conséquence inévitable de la transposition diégétique " (Palimpsestes,

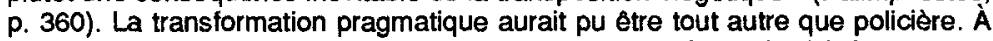
mon sens, c'est le choix du scénario policier qui transforme la diégèse, et non l'inverse; ce scénario, tout à fait absent de l'hypotexte, aurait pu être remplacé par un autre scénario tout aussi moderne. La transdiégétisation n'a pas eu comme conséquence cette transformation pragmatique du Hamlet de Shakespeare en un roman policier. 
nels, le roman de Viard et Zacharias instaure, à des niveaux différents, des lectures enchâssées. L'explicite et l'implicite se côtoient en un double contrat. D'autres problématiques de décodage textuel s'imposent, surtout pour la version modernisée d'Horatio, limogé de sa fonction de confident devant transmettre le récit véridique, et caricaturé en docteur Laurat, psychiatre. Sa fonction: lire le vécu du cas Hamlet/Kiki, effacer ses fantômes ou participer à ceux-ci. Le rôle d'Horatio s'en trouve banalisé, voire trivialisé, par son incarnation en "psy" arriviste, dont le rôle semble davantage de servir le pouvoir que d'en rendre compte. La folie de Kiki se voit donc, par l'entremise de cette modernisation diégétique (l'ami qui écoute devient le psychiatre dont on doit se payer les services), institutionnalisée, circonscrite. Oui, le Danemark est une prison, la position d'héritier également, sans doute.

Voilà une direction, une "psychologisation des profondeurs» qui aurait pu contrebalancer la myopie socio-politique que produit, dans le roman, la disparition de Fortinbras. En effet, avec le gommage d'une arrière-scène qui enracine l'action de l'hypotexte dans une perspective historico-politique (par le fait d'une transformation sémantique), les enjeux de l'hypertexte se restreignent (ou semblent se restreindre) à des rivalités sexuelles et économiques et, sous ce rapport, trivialisent le texte source, le réduisant au simple statut de fait divers crapuleux d'une certaine haute bourgeoisie française. Le genre même, le roman policier, relégué plus ou moins à tort dans la périphérie de la littérature, endosse-t-il cette trivialisation? Sommes-nous en régime sérieux? De ce Fortinbras absent, les auteurs de L'embrumé ont pourtant tenu à garder cette phrase placée en épigraphe au chapitre IX: “Oh mort! quelle fête dans ton infernale demeure! » Serait-ce là une indication de lecture forçant l'interprète-lecteur à chercher quelque part dans l'appareil politico-judiciaire l'équivalent de Fortinbras? Qui est là avant, après l'action? Qui lit Hamlet à travers ce drame familial? Le commissaire Volmand qui s'embrume petit à petit? Qui profitera de la fortune? Laurat? Laissons là ces hypothèses et risquons une interprétation qui pourrait porter sur la fusion Hamlet/Fortinbras 4 .

4 Si je ne m'abuse, les indications épigraphiques fort abondantes dans L'embrumé permettent une certaine largeur de vision à $\infty$ sujet. Le chapitre III, par exemple, illustre magistralement, par son épigraphe "Tiens! Un rat!", le parallele explicite entre les insertions en exergue de passages de Hamiet de 
44

L'occultation, considérable dans sa portée sémantique, du personnage hypotextuel Fortinbras semble donc annihiler l'effet de perspective, voire de prospective, investi dans celui-ci.

Sa disparition resitue le contexte du roman policier dans une vision volontairement myope du drame, qui renvoie peutêtre à la myopie historique contemporaine ${ }^{5}$. La profondeur de champ s'investira ailleurs sans doute. La narrativisation de l'hypotexte qu'instaurent Viard et Zacharias ne constitue pas une simple transmodalisation de type intermodale, mais aussi une lecture didactique du sujet initial, un métadiscours qui épouse le genre policier et que souligne l'allusion à l'histoire d'un procédé classique dont l'hypotexte est une des figures type: la mise en abyme.

Lorsqu'au chapitre III, Kiki/Hamlet soliloque sur son projet de crime, il place devant lui un miroir qui le déporte de sa propre image. Il parle de lui-même au deuxième degré: «Je vais être à Landru ce que Picasso est à Vélasquez." (p. 53) Suivent des allusions à des personnages de Molière, un Diafoirus du Malade imaginaire (il faudrait presque souligner deux fois le titre...) et un Trissotin des Femmes savantes. Puis, ce malade de l'imaginaire ajoute: "Moi, je vais travailler en artiste, à la Sade, à la Byron, à la Gide. " ${ }^{6}$ Comme le dirait un certain professionnel de la transtextualisation, il y a trop d'œuvres soulignées ici. "Tout masque est aussi un miroir." 7 Voilà un hypersonnage ${ }^{8}$ qui parle des contemporains de son hypotexte et qui parle de théâtre, ce qui ne gâte (n'abîme) rien; un personnage de théâtre narrativisé qui a la nostalgie de ses origines, qui lit du théâtre et qui y réagit.

Shakespeare et l'action de son palimpseste. En effet, ce troisième chapitre décrit la planification et l'exécution du meurtre de Pierre-Paul Pollon, digne calque de Polonius. C'est donc dire que ces citations ne sont pas innocentes et qu'elles ouvrent de façon manifeste d'autres pistes de lecture.

5 Les autres transformations pragmatiques dont l'effet est proximisant sont, sans doute, la chronologie, le lieu, la classe sociale, le calque modernisant du nom des personnages, les objets plus contemporains et un certain " je m'en foutisme" au niveau langagier: "Ce con de Cisco" (p. 11), par exemple. Quoique dans ce cas, il puisse bien étre question de démasquer les allusions triviales de l'hypotexte lui-même.

6 Viard et Zacharias, L'embrumé, p. 53. C'est moi qui souligne à la Gide - et pour cause, lecteur, ainsi que vous le verrez plus tard.

7 Gérard Genette, op. cit., p. 297-298, note 3.

8 Ne trouvez-vous pas qu'hypersonnage fait moins complexe d'CEdipe qu'hyperpersonnage? Bien sûr, on en perd un, mais somme toute, on ne fait qu'un motvalise. 
"Je vais être à Landru»: ces mots signalent l'identification de Kiki à un criminel notoire ${ }^{9}$ du début du siècle, à un bandit dont il proclame vouloir être le palimpseste, en quelque sorte. La singularité de cette affirmation réside dans ce mélange de morgue et de génie sur lequel se fonde le désir de l'héritier Elsen. Il veut reproduire Landru comme Picasso le fait pour Vélasquez. Ce qui n'est pas pour nous le rendre innocent, il planifie son crime en artiste, "à la Sade, à la Byron, à la Gide». Cette association Vélasquez-Gide, dans un contexte où le théâtre est sur-représenté (Molière, Les femmes savantes, Viard et Zacharias, L'embrumé, palimpseste de Hamlet de Shakespeare), ne peut que nous ramener à la petite histoire de l'identification d'un procédé littéraire identifié par André Gide et remis à l'ordre du jour par Jean Ricardou. Dans Le nouveau roman, ce dernier nous rappelle cette réflexion de Gide, tirée de son journal de 1893:

J'aime assez qu'en une œuvre d'art, on retrouve ainsi transposé, à l'échelle des personnages, le sujet même de cette œuvre. Rien ne l'éclaire et n'établit plus sûrement les proportions de l'ensemble. Ainsi, dans tels tableaux de Memling ou de Quentin Metsys, un petit miroir convexe et sombre reflète, à son tour, l'intérieur de la scène où se joue la scène peinte. Ainsi, dans le tableau des Ménines de Vélasquez (mais un peu différemment). Enfin, en littérature, dans Hamlet, la scène de la comédie [...]. Dans la Chute de la maison Usher, la lecture que l'on fait à Roderick, etc. ${ }^{10}$

Ricardou fait suivre cette citation de ce commentaire: "Notant l'analogie de cette enclave avec l'inclusion, en héraldique, d'un blason dans un autre, on se souvient que Gide propose de la nommer une mise en abyme. » 11

Avec l'allusion à Landru, nous sommes en plein palimpseste criminel: les références à Gide et à Vélasquez nous font remonter à l'histoire de la conceptualisation d'un procédé littéraire, la

9 Voici ce que dit succinctement le Dictionnaire encyclopédique Quillet à propos de cet individu: "Landru (Henri-Désiré) 1869-1922, né à Paris, criminel fameux, mort guillotine; onze victimes, toutes des femmes, lui étaient attribuées par l'acte d'accusation " (p. 3724).

10 Jean Ricardou, Le nouveau roman, Paris, Seuil, coll. "Écrivains de toujours ", 1973, p. 48-49.

11 Ibid., p. 49. 
46

mise en abyme. Quelle est donc la raison qui fait mettre Picasso au centre de ces équations "palimpsestueuses "?

Picasso/Vélasquez, deux peintres espagnols, plus précisément andalous, qui tous deux ont connu une activité artistique intense dès leur jeune âge. Picasso qui adopte, comme Vélasquez, le nom maternel. L'article de Paul Guinard, "Picasso et l'Espagne», mentionne aussi ce fait intéressant: «Et c'est le costume des Ménines que Picasso choisira pour les plus beaux portraits que Jacqueline lui ait inspirés. "12 À la fin du texte de Guinard, est reproduit un tableau, Études pour les Ménines, exécuté par Picasso en $1957^{13}$.

Cette tirade de Kiki, "Je vais être à Landru ce que Picasso est à Vélasquez», produit un effet de perspective assez particulier. Picasso fait des hyperportraits d'une œuvre d'un peintre, Vélasquez, que Gide, le rénovateur-inventeur de la mise en abyme, prend comme exemple afin d'illustrer ce procédé. Or Picasso s'est justement servi (entre autres) du tableau exemplaire cité par André Gide, Les ménines, pour "palimpsestuer" Vélasquez. Ainsi donc, le personnage central d'un roman, L'embrumé, palimpseste de Hamlet, hypersonnage d'Hamlet, investi d'une connaissance des procédés artistiques de la mise en abyme, joue son propre drame avec le génie des artistes qu'il admire. Si l'on résume le soliloque de Kiki en termes métalittéraires, il est possible de lui prêter les affirmations suivantes:

Je suis un palimpseste de génie!

Je me mets en abyme en virtuose!

Je suis l'histoire du procédé qui me met au monde!

Avec ces quelques mots, Kiki redonne à L'embrumé l'effet de perspective qui semblait être perdu avec la disparition du contexte fortinbrasien; mais il s'agit d'une perspective d'un autre ordre, de l'ordre d'un métatexte. Kiki Elsen s'identifie à un des procédés littéraires dont l'origine hypotextuelle est exemplaire: la mise en abyme, le théâtre dans le théâtre, dont La souricière dans Hamlet de Shakespeare constitue un exemple quasi canonique.

"Je serai à Landru ce que Picasso est à Vélasquez». Et je le ferai à la Gide. Je serai donc un palimpseste de la mise en

12 Paul Guinard, "Picasso et l'Espagne „, p. 81; dans Picasso, Paris, Hachette, 1967, 293 p.

13 Ibid., p. 87. 
abyme. Picasso hyperportraitise un des exemples dont se sert Gide pour mettre au jour le mécanisme de la mise en abyme et l'hypersonnage d'Hamlet s'identifie à ce lien en faisant dans l'abstrait, c'est-à-dire, jouons sur les mots, en agissant selon un mode que les autres ne peuvent se figurer. Qu'est-ce à dire encore? Qu'il est conscient du procédé qui le rend possible, lui, hypersonnage Kiki, issu d'un hypotexte dont l'un des traits exemplaires consiste en l'utilisation de la mise en abyme. Il s'affirme donc comme lecteur et interprète de ses propres projets de meurtre, projets issus d'un hypotexte qu'il s'agit de transposer; il regarde son action, sa propre reproduction comme protagoniste, il se regarde faire l'action à travers une grille artistique, littéraire, conceptualisant un procédé. Il se regarde se mettre en abyme (métatextuellement): il est un métatexte.

Avec ma palette de couleurs, de connaissances philosophiques (Kierkegaard) et littéraires, je palimpsestuerai Landru comme Picasso a modernisé Vélasquez, et ainsi que Gide traite de la mise en abyme à partir de Vélasquez et de Hamlet de Shakespeare, dont je suis moi-même une relecture. Voyez comme je représente bien ce que je re-présente.

Ainsi pourrait s'exprimer Kiki Elsen, avec l'assentiment, bien sûr, de Viard et Zacharias. Quelle est donc la terminologie genettienne ou néo-genettienne propre à traduire ce discours? Soyons ludique, voire ironique, sinon satirique. Travestissons, pastichons, chargeons, s'il le faut. Après discussion, à la Trissotin, à la Diafoirus, ne pourrions-nous pas en arriver à rendre justice à ce procédé par le terme «métanalepse»14, défini comme un discours critique qui établit à l'intérieur même de la diégèse l'histoire d'un procédé dont le texte constitue l'un des types. Ou, si vous voulez, comme un retour en arrière (l'historique d'un procédé) dont la portée est métatextuelle. Métaboliquement:

- mise en abyme, donc, mais à un niveau métatextuel, à une autre puissance;

14 Avec une certaine audace, certes, méta + analepse fusionnés en un motvalise "métanalepse". Le terme n'est pas si inélégant. Ou analepse métatextuelle: mais ne cherche-t-on pas à créer des outils conceptuels opératoires? Le seul terme "métanalepse" nous semble prendre sens, du moins dans le contexte. Métanalepse intradiégétique, où je ne m'y connais pas. 
- micro-récit qui révèle l'histoire d'un procédé qui met son propre texte au monde;

- mise en abyme, puisqu'elle révèle en miniature l'histoire de son propre procédé.

Cette tendance du roman de Viard et Zacharias à reproduire en écho son propre procédé de fabrication reçoit un éclairage des plus pertinents avec ces propos de Ricardou:

Or, si la mise en abyme peut se définir comme un narcissisme, la micro-histoire qu'elle produit, et nous retrouvons les peintres que citait Gide, est un miroir. En s'y venant mirer, I'histoire préalable risque donc d'y laisser paraître le fragment d'elle-même qu'elle a prétendu cacher. Dans une histoire qui se veut incomplète, la mise en abyme peut voir sa contestation se préciser en un pouvoir révélant. ${ }^{15}$

N'y a-t-il pas, dans L'embrumé, présence de ce narcissisme métatextuel? Ne peut-on dire que Kiki Elsen se mire dans ce micro-métadiscours et que ce dernier révèle les intentions explicites des auteurs? Viard (Henri de son prénom) préfêre "qu'on parle d'explication de texte pour définir ses livres, ou même de mise en scène; il voudrait avoir le langage d'un metteur en scène de Shakespeare qui parle familièrement à ses comédiens pour leur expliquer sans emphase comment se passent réellement les choses $\$ 16$.

Résumons-nous. Le soliloque de Kiki souligne l'existence d'une histoire métatextuelle intratextualisée, dans une lecture d'œuvre: un palimpseste. Ce que dit Kiki, c'est: «Je me représente moi-même selon des modèles types, classiques. Je m'hypotextifie en ce que je représente le processus qui m'a engendré. Je me révèle comme lecteur au second degré de mon propre agir." Une autre question se pose. N'y a-t-il pas, dans la tirade de Kiki Elsen, cette "révolte structurelle d'un fragment de récit contre l'ensemble qui le contient»17, ce qu'est avant tout la mise en abyme pour Jean Ricardou? Révolte contre le réalisme même du genre policier et peut-

15 Jean Ricardou, Problemes du nouveau roman, Paris, Seuil, coll. "Tel Quel ", 1967, p. 182.

16 Jérôme Couturier, "Viard et Zacharias. Les iconoclastes de la série noire", dans Magazine litteraire, Paris, $n^{\circ} 3$, janvier 1967, p. 43.

17 Jean Ricardou, Problèmes du nouveau roman, p. 181. 
être contre le réalisme des meurtres accomplis par d'autres que lui. Du crime effectué pour des intérêts matériels, on passe à un crime d'artiste qui proclame la gratuité de son esthétique. Du paralittéraire, on glisse dans le métalittéraire; et c'est peut-être cela, qu'avec force clins d'œil pourtant, Viard et Zacharias ont prétendu cacher.

\section{Commentaires sur le paratexte}

Les indications paratextuelles de L'embrumé traduisent, pourrait-on dire, deux contrats. D'abord celui de son autonomie comme ouvre (titre, auteurs et collection renvoyant au genre policier), mais surtout, en ce qui nous concerne, son contrat d'hypertextualité. Les épigraphes, entre autres, donnent des indications de lecture et structurent un parcours qui laisse deviner les intentions didactiques des auteurs. D'ailleurs, le mot «embrumé», petit énoncé titulaire, est déjà largement porteur du caractère ambigu de l'hypo et de lhypertexte comme de celui des personnages qu'il représente (Hamlet/Henri Elsen junior). En ce sens, il contamine le récit; factuellement, le mot "embrumé" apparaît douze fois ${ }^{18}$ dans le texte de Viard et Zacharias, juste assez pour exercer son effet contaminateur sur le personnage habituellement le plus représentatif du contrôle de soi et de la bonne logique: le commissaire de police. En clausule, les dernières paroles de Volmand osent manifester cet effet: « Je me sens un peu "embrumé”, à mon tour. »19

La façon dont Viard et Zacharias inscrivent les épigraphes questionne le lecteur. Celles des cinq premiers chapitres, par exemple, permettent, par une citation exacte, de retracer leur source dans l'hypotexte. En exergue au chapitre III, le célèbre constat, «Tiens! Un rat!», signale l'analogie de cette partie de L'embrumé avec son hypotexte; c'est dans cet épisode qu'on revoit le meurtre de Polonius, c'est-à-dire de Pierre-Paul Pollon, meurtre cette fois planifié par Hamlet/Kiki. L'épigraphe est alors identifiée comme provenant de l'acte III, scène 4.

Les épigraphes des chapitres VI, VII, VIII et IX n'ont pas reçu la marque exacte de leur origine. Rien d'innocent à cela,

18 Viard et Zacharias, op. cit., p. 80 (3 fois), p. 84 (1 fois), p. 86 (1 fois), p. 87 ( 1 fois), p. 124 ( 2 fois), p. 125 (2 fois), p. 185 ( 2 fois).

19 lbid., p. 185. 
sans doute, car, à l'instar de la symétrie reliant la fin au début de Hamlet de Shakespeare, une certaine symétrie début/fin peut être dégagée de l'hypertexte. De là, cette hypothèse, que nous aurions pu développer davantage ici, d'une correspondance entre certaines scènes des premiers chapitres et celles des derniers. D'ailleurs, comme dans l'hypotexte, Claudius/Claude Elsen ne prend vraiment l'initiative de l'action que dans la deuxième partie du roman.

Arrêtons-nous maintenant sur l'épigraphe où se manifestent les premiers aveux d'un contrat hypertextuel: "Être ou ne pas être, voici la question", signée simplement Shakespeare, sans mention exacte de l'origine de la citation 20 . Épreuve dialogique, car, juste après cette parole célèbre du Hamlet de Shakespeare qui laisse sa question sans réponse, on lit un aphorisme péremptoire cette fois: «Ne plus être, voici la réponse», signé Viard et Zacharias.

Cette réplique tient-elle déjà de la modernisation diégétique? Pas de philosophie! Une décision? Constitue-t-elle en soi une indication de parodie, de pastiche, de charge, de travestissement? Ou tout simplement une transposition en régime sérieux, avec cet élément de pragmatisme moderne propre au roman noir américain (transmodalisation intermodale, du drame (théâtre) au roman (narrativisation), dans laquelle se dessine la modernisation diégétique de l'hypotexte). Tout porte à croire que les auteurs manient le registre ironique, voire satirique, et que, tout en faisant ouvre sérieuse, ils s'amusent aux dépens de l'hypotexte, du lecteur et de la littérature en général. L'hypertexte est tout à la fois, comme son hypotexte, trivial, didactique et noble.

À propos de ce dialogue épigraphique, indice du contrat entre Viard et Zacharias et Shakespeare, ne pourrions-nous pas dire qu'il s'agit d'un pastiche, puisque le sens de la phrase «imitée» est transformé sans que le style soit véritablement modifié?

Être ou ne pas être, voici la question.

Ne plus être, voici la réponse.

Ne pourrions-nous pas parler de parodie, puisque la réponse ressemble étrangement à la «question", tout en étant symé-

20 On lit dans le roman dix épigraphes, dont cinq avec la mention exacte de la citation et cinq autres sans cette indication. 
triquement inversée? Trop d'éléments y seraient-ils changés, travestis?

Et pourquoi ne pas parler de charge: on te règle ton compte, Shakespeare, à travers un palimpseste de ton Hamlet trop souvent cité. Plus d'hésitations inutiles, plus de questions. Une affirmation. Une réponse. Mais, dans ce cas, nous glissons vers ce qui, implicitement, relève de la polémique, et le régime pourrait tout aussi bien n'être que sérieux.

Elle s'avère donc largement polysémique, cette petite conversation par-dessus les siècles.

À la page qui suit le dialogue épigraphique entre Viard et Zacharias et Shakespeare, un tableau présente les personnages et leurs interprètes (policiers), comme au théâtre. Quatorze personnages dont la moitié, exactement, reçoivent mention de leur âge, comme s'il fallait distinguer deux clans, deux catégories d'actants. Autre question que pose l'existence même de ce tableau dans le paratexte d'un roman: les autres indications (les dix épigraphes) ne suffisaient-elles pas à manifester le contrat de transposition de Hamlet de Shakespeare? Dans quel autre roman policier, en effet, peut-on lire une telle présentation de personnages? Si les auteurs se sont dit que la seule (ou la meilleure) façon d'expliquer Hamlet était de le transposer dans un roman policier moderne, pourquoi ce contrat plus théatral que romanesque? Si on lit dans Hamlet: "La scène est au Danemark » ${ }^{21}$, on découvre dans la présentation des personnages de l'hypertexte, au bas de la page cette fois: "La scène est principalement à la villa Elsen, pendant l'hiver.» Voilà bien des indications de lecture: on nous présente une pièce de théâtre dans un roman policier; plus, sans doute, qu'un roman policier tiré d'une pièce de théâtre. Un peu comme si nous étions davantage en présence d'une transformation intramodale avec narrativisation que d'un drame intermodalisé, simplement narrativisé. Dans L'embrumé, ne raconte-t-on pas des procédés de théâtre, du théâtre? Ce cadre moderne qu'est l'enquête policière ne servirait-il pas de chœur, un peu comme dans la tragédie grecque?

21 William Shakespeare, Hamlet. Le roi Lear, Paris, Gallimard, 1978, 405 p. Préface d'Yves Bonnefoy. Le tableau apparaît en p. 9 du roman. 
52

\section{À propos de mise en abyme}

Fortinbras étant éliminé, ce n'est plus le spectre de Hamletpère qu'on guette mais plutôt "le gars des lettres anonymes". Ces lettres correspondent à une certaine naturalisation (à une modernisation diégétique) du fantôme. "On m'a tué mais je ne suis pas mort" constitue sans doute le premier type de mise en abyme du roman en même temps qu'un motif obsessionnel puisqu'il apparaît dix fois dans L'embrumé. Certains propos de Ricardou nous permettent d'élargir notre conception de ce procédé:

[...] la mise en abyme n'est pas une opération nettement délimitable. Toujours se rencontre une grande diversité dans le traitement du dispositif analogique qui l'autorise. Aussi, tout ce qui se plaît, dans le texte, à établir avec quelque insistance une relation de similitude a-t-il tendance à jouer, fût-il partiel, fût-il fugace, un rôle de mise en abyme. 22

"On m'a tué mais je ne suis pas mort" articule un microrécit qui appelle l'intrigue et le dénouement, qui questionne tout autant Kiki, Claude, le docteur Laurat que le personnel de la police. Tous oscillent entre les multiples possibilités de lecture de ce texte-leitmotiv. Les auteurs donnent à lire L'embrumé, qui est une lecture de Hamlet, à travers une enquête policière qui cherche à interpréter ce billet «On m'a tué mais je ne suis pas mort", lui-même synthèse révélatrice de l'action en cours.

L'interrogatoire de Kiki par Volmand joue aussi ce rôle d'abyme, mais à une échelle plus réduite. Au chapitre IV, le roman policer prend toute sa place avec l'hypervalorisation du commissaire Volmand. Le théâtre du commissaire, à ce moment-ci, veut prendre la conscience du prince ${ }^{23}$. Il reproduit, en miniature, le meurtre joué et mis en scène par Kiki; il résume le chapitre qui précède et révèle la génialité du premier meurtre: la falsification du kilométrage, qui donne à notre Landru un alibi de premier ordre.

Au chapitre suivant, la reconstitution de l'incident "fondateur" du récit, le suicide ou pseudo-suicide de Henri Elsen (père), interroge l'hypotexte lui-même: Ophélie s'est-

22 Jean Ricardou, Le nouveau roman, p. 69.

23 Viard et Zacharias, op. cit., p. 81. 
elle vraiment suicidée? C'est sans doute cette reconstitution qui correspondrait le plus exactement au théâtre dans le théâtre tel qu'il apparaît dans Hamlet. Volmand et ses acolytes tenteront de prendre au piège les consciences des personnages: «Parfaitement! Là nous aurons le loisir d'examiner leurs réaction.» ${ }^{24}$ Ces propos de Volmand à Cornil ne sont pas sans rappeler ceux de Hamlet à Horatio. Dans ce même chapitre, suivra la visite de Kiki à sa mère: il veut mesurer l'impact produit par la scène et faire apparaître le spectre hypotextuel: "On m'a tué mais je ne suis pas mort.» 25 Même incompréhension (disons plutôt même fin de non-recevoir) de la part de Gerty/Gertrude, même éloignement, le vouvoiement: “Vous délirez» 26 , dit-elle, avec cette ignorance feinte, cette cécité volontaire (?) dont il est si difficile de mesurer l'authenticité.

Ce court survol des procédés spéculaires est loin d'être exhaustif. D'ailleurs, chaque constat de cet ordre aurait mérité d'être approfondi. Ces observations m'auront cependant permis de cerner d'un peu plus près la théorie et la pratique de quelques manifestations littéraires hypertextuelles. Dans l'ensemble de cette étude donc, je me suis efforcé d'appliquer les concepts de Gérard Genette qui concernent le phénomène de la littérature au second degré. Bien sûr, il resterait beaucoup à dire de L'embrumé comme palimpseste de Hamlet. Ainsi, par exemple, il y aurait des hypothèses intéressantes à formuler sur la symétrie entre la première (les cinq premiers chapitres) et la deuxième partie de l'œuvre. J'aurai sans doute l'occasion d'y revenir; mais serait-ce là un alibi, effet de roman policier? 